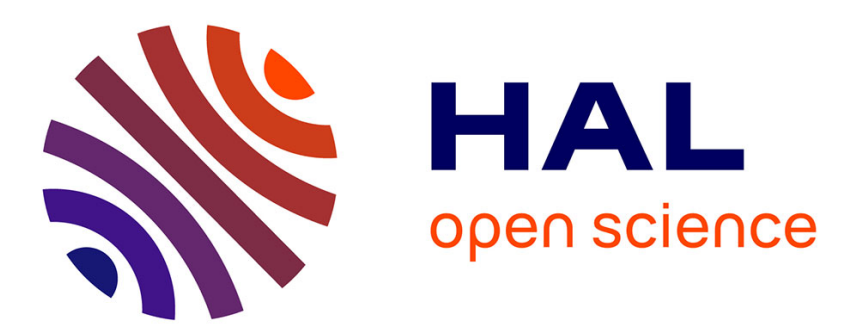

\title{
A novel cooperative platform design for coupled USV-UAV systems
}

Guangming Shao, Yong Ma, Reza Malekian, Xinping Yan, Zhixiong Li

\section{To cite this version:}

Guangming Shao, Yong Ma, Reza Malekian, Xinping Yan, Zhixiong Li. A novel cooperative platform design for coupled USV-UAV systems. IEEE Transactions on Industrial Informatics, 2019. hal02344556

\section{HAL Id: hal-02344556 https://hal.science/hal-02344556}

Submitted on 4 Nov 2019

HAL is a multi-disciplinary open access archive for the deposit and dissemination of scientific research documents, whether they are published or not. The documents may come from teaching and research institutions in France or abroad, or from public or private research centers.
L'archive ouverte pluridisciplinaire HAL, est destinée au dépôt et à la diffusion de documents scientifiques de niveau recherche, publiés ou non, émanant des établissements d'enseignement et de recherche français ou étrangers, des laboratoires publics ou privés. 


\title{
A novel cooperative platform design for coupled USV-UAV systems
}

\author{
Guangming Shao, Yong Ma, Member, IEEE, Reza Malekian, Senior Member, IEEE, Xinping Yan, and Zhixiong \\ Li, Member, IEEE
}

\begin{abstract}
This paper presents a novel cooperative USV-UAV platform to form a powerful combination, which offers foundations for collaborative task executed by the coupled USV-UAV systems. Adjustable buoys and unique carrier deck for the USV are designed to guarantee landing safety and transportation of UAV. The deck of USV is equipped with a series of sensors, and a multi-ultrasonic joint dynamic positioning algorithm is introduced for resolving the positioning problem of the coupled USV-UAV systems. To fulfill effective guidance for the landing operation of UAV, we design a hierarchical landing guide point generation algorithm to obtain a sequence of guide points. By employing the above sequential guide points, high quality paths are planned for the UAV. Cooperative dynamic positioning process of the USV-UAV systems is elucidated, and then UAV can achieve landing on the deck of USV steadily. Our cooperative USV-UAV platform is validated by simulation and water experiments.
\end{abstract}

Index Terms-USV-UAV platform. Multi-ultrasonic joint dynamic positioning algorithm. Hierarchical landing guide point generation algorithm. Cooperative positioning.

\section{INTRODUCTION}

Featured by excellent autonomy, and flexible control ability, unmanned vehicles [1], [2], including unmanned surface vehicles (USVs) [3], [4], unmanned aerial vehicles (UAVs) [5][7] have been extensively utilized in the field of engineering applications, especially in ocean and marine exploitation [4], [8], investigation, management, emergency search rescue [9], [10] for the last two decades. Compared with the manned vessels, USVs excel in executing dangerous and boring tasks

Manuscript received April 3, 2017. The authors are partially supported by the National Science Foundation of China (51579202, 51309186, 61673223), China Postdoctoral Science Foundation Funded Project (2014M560633 and 2015T80848), and Australia ARC DECRA (No. DE190100931).

G. Shao is with School of Naval Architecture Engineering, Dalian Universityof Technology, Dalian, 116024, China. (e-mail: gmshao1603@mail.dlut.edu.cn).

Y. Ma is with School of Navigation, Hubei Key Laboratory of Inland Shipping Technology, Wuhan University of Technology, Wuhan, 430063, China (Corresponding author: myongdl@whut.edu.cn).

R. Malekian is with Department of Computer Science and Media Technology, Malmö University, Malmö, 20506, Sweden, and Internet of Things and People Research Center, Malmö University, Malmö, 20506, Sweden (e-mail: reza.malekian@ieee.org).

X. Yan is with National Engineering Research Center for Water Transport Safety, Wuhan, 430063, China (email: xpyan@whut.edu.cn).

$\mathrm{Z}$. Li is with School of Engineering, Ocean University of China, Tsingdao 266100, China; and School of Mechanical, Materials, Mechatronic and Biomedical Engineering, University of Wollongong, Wollongong, NSW 2522, Australia (e-mail: zhixiong_li@uow.edu.au). continuously specially in shallow and restricted waters [4], [11]. Whereas, USVs have limited perception of a wide range of surrounding dynamic environments, and then resulting in adverse effects on their navigation safety. Meanwhile, by using an airborne camera with adjustable altitude, benefited from the flight advantages, UAVs can effectively overcome the perception deficiency of USVs. With the aid of UAVs, the highdefinition video and photos can be dynamically transmitted to the monitoring center, and then the critical departments can make sensible decisions by using above reliable information offered by UAVs. UAVs are capable of searching for quite a large range of targets [12], [13], but they do not qualify for perception of the ground and sea environments at close quarters [14]. Following that, to overcome shortcomings of each system, it is imperative that USVs system and UAVs system should be coupled together. Consequently, we focus on designing a novel cooperative USV-UAV platform for the coupled USV-UAV systems, by employing which platform the functions of each system can be consolidated and strengthened significantly.

Referring to the cooperative USV-UAV platform, the dynamic sea condition is perceived by USVs at a short range, and the large-scale maritime environment information oriented USVs can be collected by UAVs in real time. By employing the USV-UAV platform, USVs can be performed as a relay station for UAVs, information obtained by UAVs can be sent to USVs, energy refueling for UAVs can be fulfilled during the period of UAVs landing on USVs, and generous difficult tasks can be implemented by USVs and UAVs jointly [9], [11].

Following that, when cooperatively performing a task, the perspective of the coupled systems can be extended in essence, the duration of flight of UAVs can be increased substantially, and then the cooperative capacity of the coupled systems can be ameliorated markedly.

There exist excellent works on the coupled UAVs and UGVs (unmanned ground vehicles) [12], [15]. The above two platforms belongs to heterogeneous robot cooperative platforms, and owns similar task modes. However, there exist obvious differences between them when the motion models, the landing processes, and the operational safety factors are concerned. Firstly, their motion models and environments are different. There is a 6-DoFs motion model for USV, which makes USV impossible to maintain still on the dynamic water surface. 
Compared with UGV featured by 4-DoFs located on the motionless road surface, it is difficult to control the trajectory of USV accurately. Following that, owing to the disturbance brought by the dynamic water surface environment, it is rather hard to manipulate UAV landing on the carrying deck embodied on the USV with high accuracy. Whereas, motion effects of UAV on the UGV can be neglected. Secondly, their landing processes are quite different. When UAV landing on $\mathrm{UGV}$, it only needs to resolve the $2 \mathrm{D}$ positioning issue, and the pose of UGV is stable and can be controlled quite well benefited from its stable attitude. While UAV landing on USV, the motion state of USV on the water surface is time-varying, and great efforts should be paid to the 3-D positioning issue with high accuracy in the dynamic environment. Following that, their operational safety factors are different. Referring to UGV-UAV platform, UAV can stably land on the surface of UGV with high accuracy [16]. Whereas, owing to the effects brought by the 6-DoFs of USV and environment disturbance, the operation of UAV landing on USV is with relatively low safety when compared with that of the UGVUAV platform. Furthermore, after UAV landing on USV, the UAV should be locked-in in time to enhance the stability of USV-UAV platform. Consequently, due to the significantly differents between UGV-UAVs and USV-UAV, the developed platform and algorithms including RTK-GPS [17], GPS-INS [18], visual localization [16] for UGV-UAVs [16], [17] cannot be employed for resolving USV-UAVs platform under the scenario stressed in this work.

Currently, few studies address the cooperative USV-UAV platform. As stated in [9], it was the first known effort to introduce USVs into the domain of emergency response, and the first time proposed the cooperative USV-UAV systems for any domain. Results in [9] verified that USV-UAV systems played a critical role in disaster recovery. [19] established the dynamic models of AR.Drone 2.0 for four-rotor UAV and Strider v1.0 for USV. On this basis, a control structure based on a PID feedback loop is designed by using SIMULINK. Wherein the control of UAV and USV is achieved and different tasks can be performed quite well. To collect remote surface data with lowcost, [20] presented the USV and UAV assisted measurement methodology. Wherein this method adopted visual sensors located on UAV to capture high-resolution imagery, and sonar sensors deployed on USV to obtain bathymetric readings. It can be indicated that little coupling exists between USV system and UAV systems in [20]. Consequently, this paper studies the coupled systems of USV and UAV substantially, and designs the cooperative USV-UAV platform to strengthen the joint functions of USV and UAV.

The cooperative USV-UAV platform is confronted with new challenges, including the dynamic positioning of UAV related to USV, ways to generate a sequence of guide points by using which UAV can land on the deck of USV success- fully, and details of cooperative dynamic positioning process. Consequently, in this study, we illustrate the platform systems architecture, propose a multi-ultrasonic joint dynamic positioning algorithm, and demonstrate the ways to generate the intermediate guide points for UAV landing on USV in real time.

Some highlights of this paper are as follows: (1) to strengthen the advantages and overcome the shortcomings of USV system and UAV system, we design a novel cooperative platform for the coupled USV-UAV systems to form a powerful combination; (2) to maintain the stability of USV and safe shipment of UAV on the platform, we design an external adjustable buoys and unique carrier deck for the USV, by employing which the safety of landing and transportation of UAV can be guaranteed; (3) we propose a multi-ultrasonic joint dynamic positioning algorithm to achieve the position of UAV, and present a hierarchical landing guide point generation algorithm to obtain a sequence of guide points. Finally, simulation and water experiments are performed to validate the effectiveness of our cooperative USV-UAV platform.

The remainder of the paper is organized as follows. Section II states the cooperative USV-UAV platform. Section III proposes a positioning algorithm for the coupled USV-UAV systems. Section IV presents a hierarchical landing guide point generation algorithm for UAV landing on the USV. Section V plans paths represented by the cubic B-spline curves and illustrates process of UAV landing on the deck of USV. In Section VI, simulations and water experiments are deployed and analyzed. Finally, Section VII concludes this paper.

\section{BRief STATES ON COOPERATIVE USV-UAV PLATFORM}

The cooperative USV-UAV platform is a highly coupled autonomous system, and embodied with many advanced technologies, including the sensor technology, the wireless communication technology, the data process technology, and intelligent control technology. Following that, this section briefly states the systems architecture of USV, the structure composition of USV platform, the transport security design of cooperative platform, and the control systems of UAV, respectively.

\section{A. Systems architecture of USV that coupled with UAV}

Referring to the systems architecture of USV, as shown in Fig. 1, it mainly consists of the lower machine platform, and the PC platform. The low machine platform is the core controller of the USV, and is composed of the transport devices for UAV, the navigation system, the energy power system, and the sensor system. With the aid of the transport devices for UAV, the UAV can land on the deck of USV safely. The navigation system, including GPS and the vision system, offers the information for positioning. The energy power system consists of the USV rudder system, the USV 

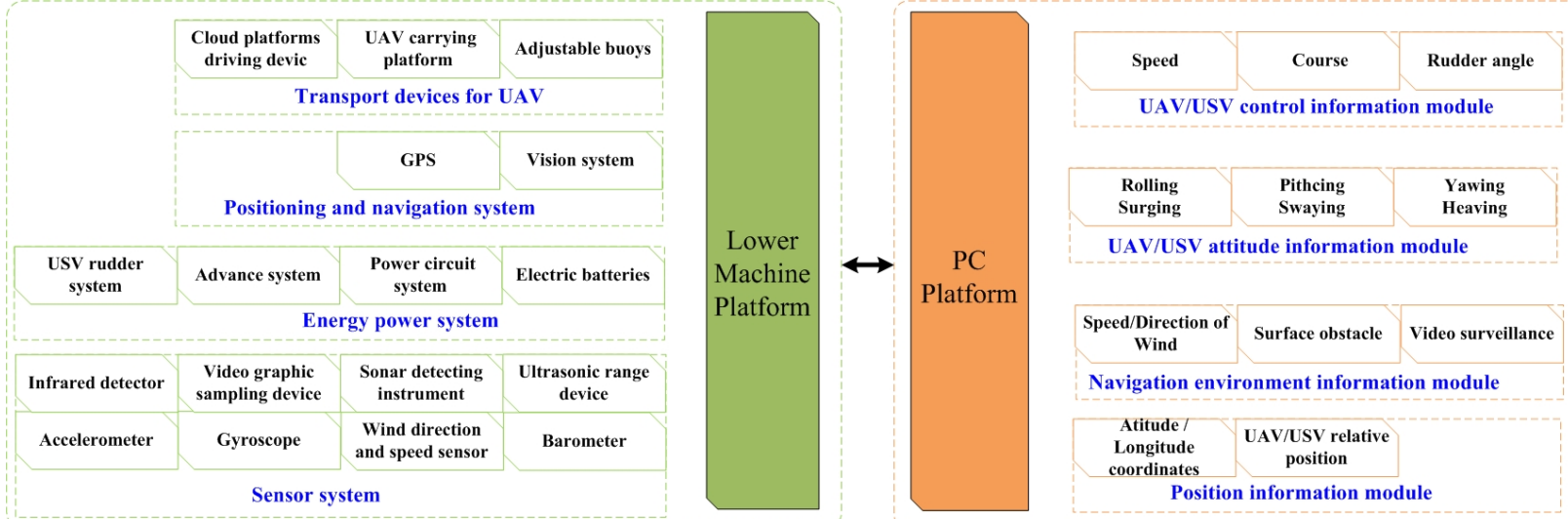

Fig. 1. Systems architecture of the USV platform that coupled with UAV

advance system, the power circuit system, and the electric batteries. Real time data can be obtained by the sensor systems. The PC platform is the control terminal of the cooperative USV-UAV platform, consisting of USV control information module, attitude information module, navigation environment information module, and the position information module.

The lower machine platform receives the data obtained by the sensor system, and processes above data and sends to the PC platform via the communication module. According to the USV's motion state, the PC platform sends corresponding commands to the lower machine platform. Following that, within the lower machine platform, the commands are analyzed and transmitted to the energy power system, and then the USV can be manoeuvred when executing those commands. Meanwhile, as the control terminal, the PC platform displays all the information and plays the role of a surveillance center. Details of USV's system architecture can be referred to [21].

Referring to UAV in the coupled USV-UAV systems, it should be with a small overall size, light weight, a high efficiency motor, and can be equipped with lots of precision instruments. Meanwhile, to achieve excellent maneuverability, UAV should be with stability control system, by employing which the position, the height, the attitude and the velocity of the UAV can be commanded quickly and conveniently. Consequently, due to the four rotor UAV is characterized by above advantages, we select the four rotor UAV as the potential UAV in our cooperative USV-UAV platform. Following that, the UAV can execute generous of tasks with the cooperation of USV.

\section{B. Structure composition of the USV platform}

When it comes to the modular structure composition shown in Fig. 2, it promotes the solid cooperation among all parts of the USV platform. The adjustable buoys enhance the stability of the hull when UAV landing on the USV platform, the double differential water-jet propulsion systems improve the manoeuvrability of USV and qualify for the navigation under severe environment, and the retractable UAV carrying platform extends the deck area and fulfills the deck utilization efficiency of USV. It can be indicated that, the USV platform is featured by a fast sailing speed, an excellent environmental adaptability, a quite complete control system that equipped with a variety of sensor devices. Consequently, the above USV platform would play a critical role in the coupled USV-UAV systems.

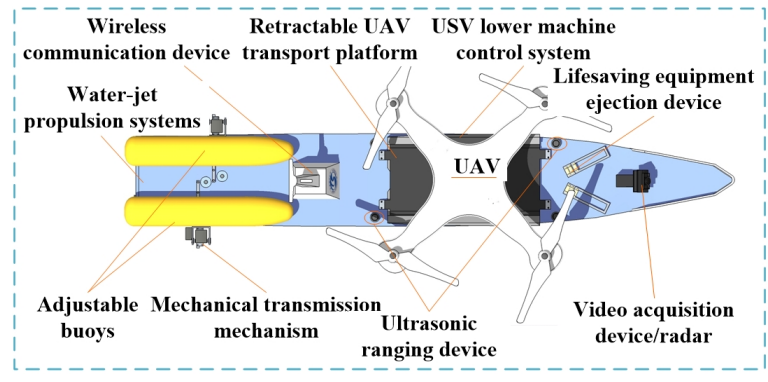

Fig. 2. Structure composition of the coupled USV-UAV platform.

\section{Transport security design of USV for UAV}

To fulfill the goals of UAV landing on the deck of USV, this section details the transport security design of USV. It is significant to consider the adverse effects existing in the coupling process between UAV and UAV. The adverse effects include two parts, one is that the motion of USV in the voyage is in the form of six-degree-of-freedom, and the other is that airflow would be generated by the rotor of UAV during its flying period. Resulting from the adverse effects brought by the first part, it is challenging for UAV landing on the deck of the USV accurately. Referring to the second part, airflow would disturb the stability of the USV remarkably. Consequently, to guarantee UAV land on the deck of USV under the adverse maritime circumstances, it is necessary to extend the available deck area for UAV landing substantially, and it would be expedient to design some novel device by utilizing which UAV can be fixed on the deck of USV efficaciously.

The above issues can be essentially handled by employing the retractable UAV carrying platform as shown in Fig. 3. The landing area of UAV is denoted as a capital letter $\mathrm{H}$ on the 


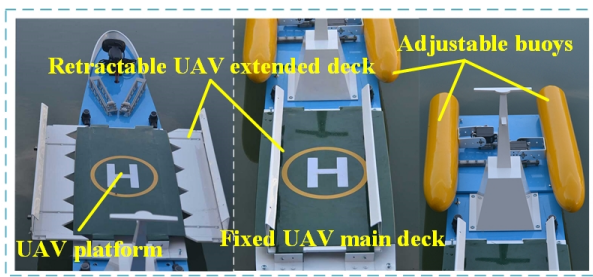

Fig. 3. Retractable UAV carrying platform that embedded in USV

fixed deck of USV, the retractable decks with guardrail and air vents are bilateral symmetrically emplaced on each side of the fixed deck. With the extended landing deck area, UAV can successfully land on the deck of USV with high probability. The deck should be contracted when UAV loaded on the deck of USV. Following that, UAV can be fixed tightly, and the deck area of USV can be cut substantially. Consequently, with the design of our retractable UAV carrying platform, the transport security of UAV can be achieved substantially.

Furthermore, the adjustable buoys of USV are shown in the right part of Fig. 3. The buoys in the spread state can enhance the large angle stability of USV during UAV landing period. To ensure that the speed of USV is not affected, the buoys are in the folded state if UAV landed on the deck of USV, the resistance of the USV encountered can be reduced noticeably during USV navigation period.

\section{Dynamic Positioning for COUPLED USV-UAV Systems}

To fulfill the cooperation between USV system and UAV system at close quarters, it is crucial to achieve the position of UAV in real time. Following that, we propose a multiultrasonic joint dynamic positioning algorithm, and then the dynamic position of UAV can be reached.

Fig. 4 illustrates the side view of USV's navigation attitude, and the body-fixed coordination system $O_{S}-X_{B S} Y_{B S} Z_{B S}$ (noted as $F_{B S}$ ) of USV with the origin $O_{S}(0,0,0)$. Within $F_{B S}$, $O_{S} X_{B S}, O_{S} Y_{B S}$, and $O_{S} Z_{B S}$ denote the longitudinal axis, the transversal axis, and the normal axis, respectively. Directions of $O_{S} X_{B S}, O_{S} Y_{B S}$, and $O_{S} Z_{B S}$ are from aft to fore, toward to starboard, are upward from bottom to top, respectively. $\phi_{u s v}$, $\theta_{u s v}$, and $\psi_{u s v}$ represent the corresponding rotation angles of $O_{S} X_{B S}, O_{S} Y_{B S}$, and $O_{S} Z_{B S}$ in sequence, and denote the rolling angle, the pitching angle, and azimuth of USV, respectively [22]. As shown in Fig. 3 and Fig. 4, the front deck of USV is equipped with four sensors, two sensors are arranged on the port side, and the other two symmetrically arranged on the starboard side.
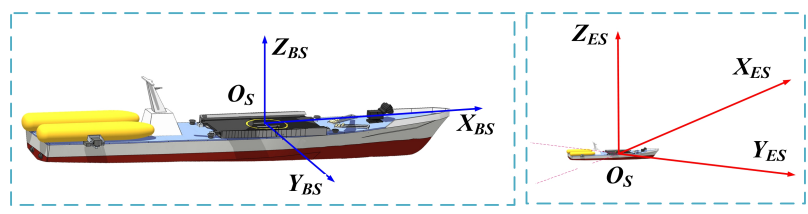

Fig. 4. Body fixed coordination system $F_{B S}$ and earth fixed coordination system $F_{E S}$ of coupled USV-UAV systems
Within our positioning algorithm, the distances between UAV and multiple ultrasonic sensors that loaded on USV can be detected by employing time of arrival (TOA) [23], and then the position of UAV relative to USV in the body-fixed coordination system $F_{B S}$ of USV can be calculated by utilizing above distances, and the real-time position of UAV in the positioning coordinate system can be obtained by employing technology of coordinate transform.

\section{A. ToA employed in cooperative USV-UAV platform}

Referring to ToA positioning solutions, distances between devices are realized based on the transmit time of an radiofrequency wave between sender and receiver [23]. During the period of UAV landing on the deck of USV, it is necessary to determine the position of the UAV relative to USV. Owing to UAV's locating equipment on the USV would move with USV in six freedoms, while the wide-beam ultrasonic sensor can adapt to the complex and practical environment perfectly,and then it is sensible that wide-beam ultrasonic sensors are selected for measuring the distances between UAV and USV.

Suppose $D, c$ and $t$ are the distance between UAV and the sensor, the transmit velocity of ultrasonic sensor, and the time from sender to receiver, respectively. Meanwhile, units of $D$, $c$ and $t$ are $m, m / s$, and $s$, respectively. Then, the distance $D$ using ToA can be $D=\frac{c t}{2}$.

\section{B. Multi-ultrasonic joint dynamic positioning algorithm}

Within our multi-ultrasonic joint dynamic positioning algorithm, there are four ultrasonic sensors are utilized to determine distances between USV and UAV based on ToA, respectively, and then after a set of computations and coordination system transformation, finally the dynamic position of UAV relative to USV can be achieved.

Suppose the position of UAV can be $X=\left[\begin{array}{lll}x & y & z\end{array}\right]^{T}$, the positions of the four sensors equipped on USV are $X_{s i}=$ $\left[\begin{array}{lll}x_{s i} & y_{s i} & z_{s i}\end{array}\right]^{T}, i=1, \ldots, 4$, respectively. We set deck origin of USV is located on $O_{S}(0,0,0)$, then the distance $d_{s i}$ between each sensor and the deck origin $O_{S}$ can be $d_{s i}=\sqrt{x_{s i}^{2}+y_{s i}^{2}+z_{s i}^{2}}$, and the slant distance $r_{s i}$ between UAV and each sensor located on USV can be $r_{s i}=\sqrt{\left(x-x_{s i}\right)^{2}+\left(y-y_{s i}\right)^{2}+\left(z-z_{s i}\right)^{2}}$, where $i=1, \ldots, 4$. The distance between UAV and deck origin of USV denoted as $r_{u u}$ can be $r_{u u}=\sqrt{x^{2}+y^{2}+z^{2}}$.

For each $r_{s i}$ after expanded, it can be

$$
\begin{aligned}
r_{s i}^{2} & =\left(x-x_{s i}\right)^{2}+\left(y-y_{s i}\right)^{2}+\left(z-z_{s i}\right)^{2} \\
& =r_{u u}^{2}+d_{s i}^{2}-2\left(x x_{s i}+y y_{s i}+z z_{s i}\right) \quad i=1, \ldots, 4 .
\end{aligned}
$$

When it comes to Eq. (1), it is obvious that the locations of the four sensors $X_{s i}(i=1, \ldots, 4)$ are known beforehand, and then the distance $d_{s i}$ between each sensor and the deck origin $O_{S}$ can be known in advance. The value of $r_{s i}(i=1, \ldots, 4)$ can be achieved by the sensors, and we only target at obtain the position of UAV $X=\left[\begin{array}{lll}x & y & z\end{array}\right]^{T}$. After deleting $r_{u u}$, 
suppose $v_{i j}=0.5\left[\left(r_{s i}^{2}-r_{s j}^{2}\right)-\left(d_{s i}^{2}-d_{s j}^{2}\right)\right](i, j=1, \ldots, 4, i \neq j)$, and $V=\left[\begin{array}{llllll}v_{12} & v_{13} & v_{14} & v_{23} & v_{24} & v_{34}\end{array}\right]^{T}$, then the following equations are reached as below.

$$
\begin{aligned}
& \left(x_{s 2}-x_{s 1}\right) x+\left(y_{s 2}-y_{s 1}\right) y+\left(z_{s 2}-z_{s 1}\right) z=v_{12} \\
& \left(x_{s 3}-x_{s 1}\right) x+\left(y_{s 3}-y_{s 1}\right) y+\left(z_{s 3}-z_{s 1}\right) z=v_{13} \\
& \left(x_{s 4}-x_{s 1}\right) x+\left(y_{s 4}-y_{s 1}\right) y+\left(z_{s 4}-z_{s 1}\right) z=v_{14} \\
& \left(x_{s 3}-x_{s 2}\right) x+\left(y_{s 3}-y_{s 2}\right) y+\left(z_{s 3}-z_{s 2}\right) z=v_{23} \\
& \left(x_{s 4}-x_{s 2}\right) x+\left(y_{s 4}-y_{s 2}\right) y+\left(z_{s 4}-z_{s 2}\right) z=v_{24} \\
& \left(x_{s 4}-x_{s 3}\right) x+\left(y_{s 4}-y_{s 3}\right) y+\left(z_{s 4}-z_{s 3}\right) z=v_{34}
\end{aligned}
$$

It can be seen in Eq. (2-7) that, $v_{i j}$ is known in advance, where $i, j=1, \ldots, 4, i \neq j$. Suppose the coefficient matrix of Eq. (2-7) is $A_{\text {all }}$, and its value also can be known beforehand. Then,

$$
A_{\text {all }} X=V
$$

Taking into account the position distribution of the four ultrasonic sensors on the deck of USV, to achieve the dynamic position of UAV from Eq. (8), it is feasible to obtain resolutions by utilizing the data collected by any three of four sensors, and the data of the remainder sensor can be employed for verify the effectiveness of the coupled USV-UAV systems. Consequently, we randomly select any three sensors, and the simplified coefficient matrix $A_{3 o 4}$ of $X$ can be

$$
A_{3 o 4}=\left[\begin{array}{lll}
x_{s 2}-x_{s 1} & y_{s 2}-y_{s 1} & z_{s 2}-z_{s 1} \\
x_{s 3}-x_{s 1} & y_{s 3}-y_{s 1} & z_{s 3}-z_{s 1} \\
x_{s 3}-x_{s 2} & y_{s 3}-y_{s 2} & z_{s 3}-z_{s 2}
\end{array}\right]
$$

Due to the four sensors are loaded on the deck of USV with the same height above the waterline, then the value of $z_{s i}$ can be 0 , and the value of $z_{s i}-z_{s j}$ equals to 0 , where $i, j=1, \ldots, 4, i \neq j$. Then,

$$
A_{3 o 4}^{\prime}=\left[\begin{array}{ll}
x_{s 2}-x_{s 1} & y_{s 2}-y_{s 1} \\
x_{s 3}-x_{s 1} & y_{s 3}-y_{s 1} \\
x_{s 3}-x_{s 2} & y_{s 3}-y_{s 2}
\end{array}\right]
$$

Consequently, after resolving the following Eq. (11), the horizontal position of UAV $X_{h}=\left[\begin{array}{ll}x & y\end{array}\right]^{T}$ can be reached.

$$
A_{3 o 4}^{\prime} X_{h}=\left[\begin{array}{l}
v_{12} \\
v_{13} \\
v_{23}
\end{array}\right]
$$

With the reached $X_{h}=\left[\begin{array}{ll}x & y\end{array}\right]^{T}, \forall i=1, \ldots, 4$, the height of UAV $z$ can be $z=\sqrt{r_{s i}^{2}-d_{s i}^{2}-x^{2}-y^{2}+2\left(x x_{s i}+y y_{s i}\right)}$.

Finally, the dynamic position of UAV $X=\left[\begin{array}{lll}x & y & z\end{array}\right]^{T}$ can be achieved by using our multi-ultrasonic dynamic positioning algorithm.

As presented in Fig. 4, $Y_{B S} O_{S} Z_{B S}$ is a in-plane of the bodyfixed coordination system $F_{B S}$ of USV, and $Y_{E S} O_{S} Z_{E S}$ is a inplane of the earth fixed coordination system $O_{S} X_{B S}, O_{S} Y_{B S}$ (noted as $F_{E S}$ ) of USV. It can be seen that, after a series of motion transformation, the position of USV in $F_{B S}$ system can be transferred to the position in $F_{E S}$ system.

Suppose $X^{\prime}=\left[\begin{array}{lll}x^{\prime} & y^{\prime} & z^{\prime}\end{array}\right]^{T}$ is the position of UAV in $F_{E S}$ coordination system, and $T_{3 D}$ is the transformation matrix from $F_{B S}$ to $F_{E S}$ and can be $T_{3 D}=T_{3 d x} \cdot T_{3 d y} \cdot T_{3 d z}$, where $T_{3 d x}, T_{3 d y}$, and $T_{3 d z}$ are the matrices corresponding to $O_{S} X_{B S}$, $O_{S} Y_{B S}$, and $O_{S} Z_{B S}$, respectively. The expressions of $T_{3 d x}, T_{3 d y}$, and $T_{3 d z}$ can be

$T_{3 d x}=\left[\begin{array}{ccc}1 & 0 & 0 \\ 0 & \cos \phi_{u s v} & \sin \phi_{u s v} \\ 0 & -\sin \phi_{u s v} & \cos \phi_{u s v}\end{array}\right] \quad T_{3 d y}=\left[\begin{array}{ccc}\cos \theta_{u s v} & 0 & \sin \theta_{u s v} \\ 0 & 1 & 0 \\ -\sin \theta_{u s v} & 0 & \cos \theta_{u s v}\end{array}\right]$

$$
T_{3 d z}=\left[\begin{array}{ccc}
\cos \psi_{u s v} & -\sin \psi_{u s v} & 0 \\
\sin \psi_{u s v} & \cos \psi_{u s v} & 0 \\
0 & 0 & 1
\end{array}\right]
$$

Within $T_{3 d x}, T_{3 d y}$, and $T_{3 d z}, \phi_{u s v}, \theta_{u s v}$, and $\psi_{u s v}$ denote the corresponding rotation angles of $O_{S} X_{B S}, O_{S} Y_{B S}$, and $O_{S} Z_{B S}$, respectively. Consequently, $X^{\prime}$ can be $X \cdot T_{3 D}$, and $X^{\prime}=X \cdot T_{3 d x}$. $T_{3 d y} \cdot T_{3 d z}$.

Algorithm Multi - ultrasonic joint dynamic positioning

Input: Positions $X_{s i}$ of the $i$ th sensors, $i=1, \ldots, 4$; slant distance $r_{s i}(i=1, \ldots, 4)$ between UAV and the $i$ th sensor; rolling angle $\phi_{u s v}$, pitching angle $\theta_{u s v}$, and azimuth $\psi_{u s v}$ of USV.

Output: dynamic position $X^{\prime}$ of UAV.

Parameters: distance $d_{s i}$ between the $i$ th sensor and deck origin $O_{S}$; sensor combination $A_{i o j}^{\prime}$; Initial position $X$ of UAV; intermediate variable $v_{i j}$ and matrix $V$.

1 Initial $r_{s i}, \phi_{u s v}, \theta_{u s v}$, and $\psi_{u s v}$.

2 Calculate $d_{s i}$ and $r_{s i}, i=1, \ldots, 4$.

3 Foreach $i \in\{1, \ldots, 4\}$ Do

4 Foreach $j \in\{1, \ldots, 4\}$ Do

$5 \quad$ If $i \neq j$ Then

$6 \quad$ Calculate $v_{i j}=0.5\left[\left(r_{s i}^{2}-r_{s j}^{2}\right)-\left(d_{s i}^{2}-d_{s j}^{2}\right)\right]$;

\section{End If}

8 End Foreach

9 End Foreach

10 Update $V=\left[\begin{array}{llllll}v_{12} & v_{13} & v_{14} & v_{23} & v_{24} & v_{34}\end{array}\right]^{T}$.

11 Get $A_{3 o 4}^{\prime}$ by Eq.(10)

12 Calculate $X_{h}=\left[\begin{array}{ll}x & y\end{array}\right]^{T}$ by Eq.(11).

12 Calculate $z=\sqrt{r_{s i}^{2}-d_{s i}^{2}-x^{2}-y^{2}+2\left(x x_{s i}+y y_{s i}\right)}$.

13 Get $X=\left[\begin{array}{lll}x & y & z\end{array}\right]^{T}$.

14 Calculate $T_{3 d x}, T_{3 d y}$, and $T_{3 d z}$ by Eq.(12)-(13).

15 Get $X^{\prime}=X \cdot T_{3 d x} \cdot T_{3 d y} \cdot T_{3 d z}$.

IV. Hierarchical LaNDing GUIDE POINT GENERATION ALGORITHM FOR UAV LANDING ON THE USV

The landing guide points are crucial for UAV landing on the USV. By utilizing a sequence of guide points, the UAV 
would land on its carrying platform successfully. Each guide point should fall in the range of the ultrasonic sensors. Limited by the range of the ultrasonic sensors, to guarantee the UAV smoothly lands on the USV, it is reasonable to set a series of guide points with a certain decreasing height. Meanwhile, the direction of the guide points should be toward the center of the deck of the USV, and then the guide points would be detected by the UAV all the time. Following that, take into account above considerations, we propose a hierarchical landing guide point generation algorithm for UAV landing on the deck of USV.

Fig. 5 shows the schematic of guide points generated for UAV in the earth-fixed coordinate system $F_{E S}$. When UAV flies in the range of ultrasonic sensors, the position $P_{O S \_A}\left(x^{\prime}, y^{\prime}, z^{\prime}\right)$ in Fig. 5(a) and Fig. 5(b) of the UAV can be sensed by the ultrasonic sensors in time. Within our proposed algorithm, $P_{O S}{ }_{A}\left(x^{\prime}, y^{\prime}, z^{\prime}\right)$ is selected as the original guide point, and the landing position $G\left(0,0, h_{G}\right)$ is set as the last guide point of UAV, where $h_{G}$ is the height of the UAV landing platform above the waterline of USV.

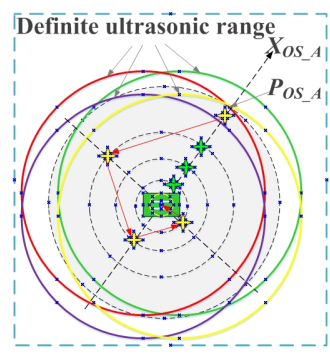

(a) Vertical view

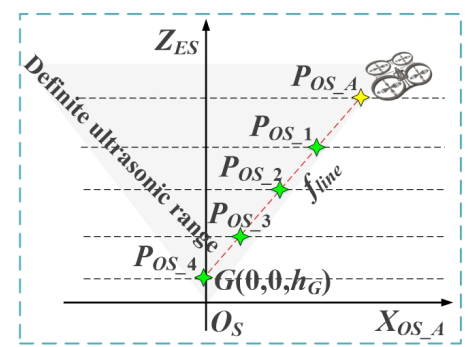

(b) Guide points generation schematic.
Fig. 5. Schematic of guide points generated for UAV in coordinate system $F_{E S}$.

Suppose there are $m$ guide points should be generated by our algorithm, and the positions of the guide points $P_{O S_{-} i}$ $(i=1, \ldots, m)$ are distributed on the line that connected $P_{O S \_A}\left(x^{\prime}, y^{\prime}, z^{\prime}\right)$ with $G\left(0,0, h_{G}\right)$. As shown in Fig. 5(b), there are $m=4$ sequential guide points $P_{O S_{-} i}(i=1, \ldots, 4)$, and all of them are on the line $\overline{P_{O S_{-}{ }_{A} P_{O S} 4}}$, where $P_{O S_{-} 4}$ and $G\left(0,0, h_{G}\right)$ coincide with each other. The line function $f_{\text {line }}$ can be

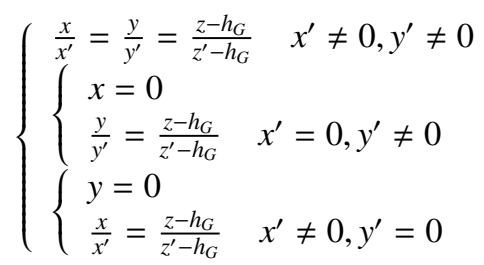

Following that, after employing the known position $P_{O S \_A}\left(x^{\prime}, y^{\prime}, z^{\prime}\right)$ sensed by ultrasonic sensors, the landing position $G\left(0,0, h_{G}\right)$ highlighted in Fig. 5(b), and the line function $f_{\text {line }}$ in Eq. (14), we can obtain a sequence of $m$ guide points, where $m=4$ in Fig. 5(b). The following equation can generate guide points distributed on the line $\overline{P_{O S_{-}{ }_{A} P_{O S} 4}}$ in the $F_{E S}$ system, where $P_{O S}^{x}, P_{O S}^{y}$, and $P_{O S i}^{z}$ denote the coordinates in three-dimension space of $P_{O S} i(i=1, \ldots, m)$, respectively.

$$
\left\{\begin{array}{l}
P_{O S_{-} i}^{x}=k_{i} x^{\prime} \\
P_{O S_{-i}}^{y}=k_{i} y^{\prime}, \quad k_{i}=\frac{P_{O S i}^{z} h_{G}}{z^{\prime} h_{G}} \\
P_{O S_{-i} i}^{z}=h_{G}+\frac{(m-i) \cdot\left(z^{\prime}-h_{G}^{\prime}\right)}{m}
\end{array}\right.
$$

Meanwhile, as shown in Fig. 5, to ensure a safe and smooth landing, the generated $m$ guide points should be equally allocated to the range of $[0,2 \pi]$. Then, for $m$ levels, the UAV should cover the arc with the radius of $P_{O S}^{x}{ }_{i}$ centered on the carrying platform for UAV. As illustrated in Fig. 5(a), for $i=1, \ldots, m$, the guide points are in yellow color and can be

$$
P_{O S_{-} i}=P_{O S_{-} i} \cdot\left[\begin{array}{ccc}
\cos \frac{2 \pi i}{m} & -\sin \frac{2 \pi i}{m i} & 0 \\
\sin \frac{2 \pi i}{m} & \cos \frac{2 \pi i}{m} & 0 \\
0 & 0 & 1
\end{array}\right]
$$

Finally, by utilizing above generated sequential guide points, the UAV would effectively follow the guide points and then land on the carrying platform safely and smoothly.

\section{Path PLANNing For UAV Landing on USV and Coupling PROCESS FOR USV-UAV SYSTEMS}

\section{A. Three-dimensional path planning for UAV landing on USV}

After generating a sequence of guide points for UAV, it is significant to plan effective paths in three-dimensional space for UAV landing on its carrying platform on the USV by employing the above guide points. Referring to the UAV path planning problems, many techniques have been introduced to plan paths for UAV including [1], [5], [6]. The cubic B-spline curve is typically utilized to represent the paths for UAV. Within the cubic B-spline curves, the velocity variation and acceleration variation of USV are continuous, there are few changes in curvature, and there exist no singular points.

Suppose there are $l+n+1$ space vertexs $P_{i}(i=0,1, \ldots, l+n)$, $l$ is the number of the curve segments, $n$ is the index of the curve. The path of UAV is denoted as $L_{U A V}$ and consists of $k$ curve segments $L_{k, n} . L_{k, n}$ is controlled by the control point $P_{i}(k=0,1, \ldots, l, i=0,1, \ldots, l+n)$. Then, for $k=0,1, \ldots, l$, the $k$ th curve segment with index $n$ can be $L_{k, n}(t)=\sum_{i=0}^{n} P_{i+k} G_{i, n}(t) \quad t \in[0,1]$, where $G_{i, n}(t)$ is the basis function of $L_{k, n}$, and can be $G_{i, n}(t)=\frac{1}{n !} \sum_{j=0}^{n-i}(-1)^{j} C_{n+1}^{j}(t+n-$ $i-j)^{n}$.

Then, referring to the cubic B-spline, its curve index $n=3$, and its curve segment $L_{k, 3}(t)(k=0, t \in[0,1])$ can be and its basic curves are shown in Eq. (17).

$$
L_{0,3}(t)=\frac{\left[\begin{array}{llll}
1 & t & t^{2} & t^{3}
\end{array}\right]}{6}\left[\begin{array}{cccc}
1 & 4 & 1 & 0 \\
-3 & 0 & 3 & 0 \\
3 & -6 & 3 & 0 \\
-1 & 3 & -3 & 1
\end{array}\right]\left[\begin{array}{l}
P_{0} \\
P_{1} \\
P_{2} \\
P_{3}
\end{array}\right]
$$

It shows that, as $k$ increased, the sequence of control points $\left[\begin{array}{llll}P_{k} & P_{k+1} & P_{k+2} & P_{k+3}\end{array}\right]^{T}$ would be shifted correspondingly, 
where $k=0,1, \ldots, l$. And then, for each $k(k=0,1, \ldots, l)$, $L_{k, 3}$ can be generated in succession. Finally, the path of UAV $L_{U A V}$ can be achieved continuously.

Furthermore, when it comes to the details of $L_{U A V}$, its cubic $B$-spline curve should pass through the initial sensed point $P_{O S_{-} A}$ and the final landing point $P_{O S_{-} m}$. Following that, to satisfy above requirements, for the point $P_{O S_{-} A}$, we add another two control points $P_{a d d \_1}$ and $P_{a d d \_2}$, where the three points overlap each other. And for $P_{O S_{-} m}$, we also add two control points $P_{a d d_{-} m_{-} 1}$ and $P_{a d d_{-} m_{-} 2}$, where the three points overlap each other. Consequently, the two points $P_{O S_{-} A}$ and $P_{O S_{-} m}$ would fall on the cubic B-spline curve of the UAV.

cubic B-spline curves are planned for the UAV. Consequently, with the aid of the curves, the UAV would proceed in the range of ultrasonic sensors during the landing period, and then smoothly land on its carrying platform smoothly and steadily. Finally, to fix the UAV tightly, the carrying platform would be folded once the UAV landed successfully.

\section{SimULATIONS AND EXPERIMENTS}

To validate the effectiveness of the coupled USV-UAV systems, we carry out a series of simulation and experiments. Suppose $L_{O A}, B_{M}, D_{M}, d_{r M}$ denote USV's parameters length overall, moulded breadth, depth, and draft in sequence, where Algorithm Hierarchical landing guide point generation algorithm $L_{O A}=165.1 \mathrm{~cm}, B_{M}=13.2 \mathrm{~cm}, D_{M}=15 \mathrm{~cm}$, and $d_{r M}=11 \mathrm{~cm}$.

Input: UAV initial capture position and the original guide point $P_{O S \_}\left(x^{\prime}, y^{\prime}, z^{\prime}\right)$; the height $h_{G}$ of the UAV landing platform above the waterline of USV; the number of guide points $m$.

Output: three-dimensional path set $L_{0,3}(t)$ denoted by cubic B-spline for UAV landing on USV.

Parameters:line function $f_{\text {line }}$; guide points $P_{O S} i$.

1 Calculate $f_{\text {line }}$ with $P_{O S_{-} A}\left(x^{\prime}, y^{\prime}, z^{\prime}\right)$ by Eq. (14).

2 Calculate $P_{O S} i$ by Eq. (15).

3 Get average distribution of $P_{O S_{-} i}$ by Eq. (16).

4 Update $P_{\mathrm{OS}_{-} i}$ of UAV.

5 Calculate $L_{0,3}^{-}(t)$ by Eq. (17).

6 Get path set $L_{0,3}(t)$ for UAV landing on USV.

\section{B. Coupling process for the USV-UAV systems}

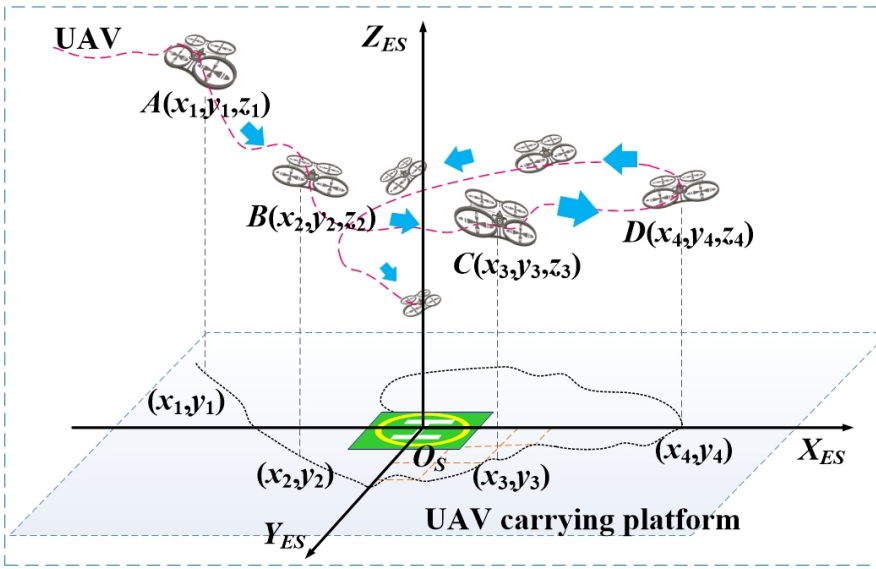

Fig. 6. Schematic of the coupling process of the USV-UAV systems that the UAV descends to the deck of the USV.

As shown in Fig. 6, in the $F_{E S}$ coordination system, when the UAV enters into the range of ultrasonic sensors at the position ' $A$ ', a sequence of guide points ' $B$ ', ' $C$ ', ' $D$ ', $\ldots$ would be generated for UAV based on the hierarchical landing guide point generation algorithm. With the above guide points, the

\section{A. Simulation}

When entering the range of ultrasonic sensors, the initial position $P_{O S \_A}$ of UAV is captured in the earth-fixed coordination system $F_{E S}$. Suppose $P_{O S \_}=(300,400,1000)$, the final landing position on the USV deck is $G(0,0,50)$, where $h_{G}=50$. And there are $m=10$ guide points would be generated for the UAV landing on its carrying platform.

Following that, by employing the hierarchical landing guide point generation algorithm elucidated in Section IV, the threedimensional hierarchical guide points $P_{O S_{-} i}(i=1, \ldots, 10)$ for the UAV landing on its carrying platform are shown in Table I.

TABLE I

REACHED 3D HIERARCHICAL GUIDE POINTS GENERATED FOR UAV (CM)

\begin{tabular}{|c|c|c|c|c|c|c|c|c|c|c|c|}
\hline & $P_{O S}$ & $P_{O S} 1$ & $P_{O S} 2$ & $P_{O S} 3$ & $P_{O S}$ & $P_{\text {OS } 5}$ & $P_{O S} 6$ & $P_{O S} 7$ & $P_{O S}$ & $P_{O S}$ & $P_{O S}$ \\
\hline & 300 & $430 . \overline{04}$ & $378 . \overline{5} 0$ & $201 . \overline{40}$ & $-4 . \overline{55}$ & $-15 \overline{0}$ & $-19 \overline{1}$ & $-14 \overline{2}$ & $-57 . \overline{54}$ & $0.7 \overline{6}$ & 0 \\
\hline & 400 & 132.54 & -129.37 & -286.25 & -300 & -200 & -59 & 48.51 & 81.78 & 50 & 0 \\
\hline & 1000 & 905 & 810 & 715 & 620 & 525 & 430 & 335 & 240 & 145 & 50 \\
\hline
\end{tabular}

According to the results in Table I, the generated guide points can be treated as the control points $P_{i}$ in the UAV path planning algorithm, wherein $P_{0}=P_{O S_{-} A}$, and $P_{i}=P_{O S_{-} j}$, where $i=0,1, \ldots, 9, j=i+1$. As stated in Section V-A, to guarantee the UAV pass through $P_{O S_{-}{ }_{A}}$ and $P_{O S_{-} m}$, control points $P_{\text {add }} 1$ and $P_{\text {add } 2} 2$ are added at the initial of the UAV path, and another two control points $P_{a d d_{-} m_{-} 1}$ and $P_{\text {add }{ }_{-} m_{-} 2}$ are added at the final of the UAV path, where $m=10$. Consequently, there are 15 control points.

Due to there are 15 control points, by subtracting three control points, then the number of the path sections is 12 . For the $i$ th path section, by using $P_{i}, P_{i+1}, P_{i+2}$, and $P_{i+3}(i=$ $0,1, \ldots, 11)$, the cubic B-spline curve can be obtained. Then, after iteration for 12 times till the last path section reached, the UAV path planning process would be completed. Fig. 7 shows the planned paths for the UAV in three-dimensional space, and the corresponding positions of the UAV carrying platform are highlighted in color lines. 


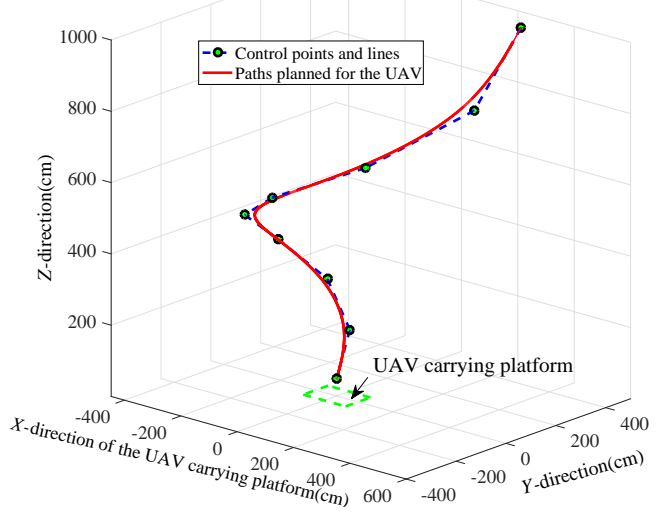

Fig. 7. Planned paths in three-dimensional space for the UAV.

\section{B. Experiments}

Our designed cooperative USV-UAV platform has won special awards of Chinese Ocean Vessel Design and Model Making Competition in 2015 and 2017. Details can be referred to [4]. Experiments are carried out in the range of $70 \mathrm{~m} \times 70 \mathrm{~m}$ water area as shown in Fig. 8, and wave height falls in $[0.1 \mathrm{~m}$, $0.3 \mathrm{~m}]$. The unit of length is centimeter system.

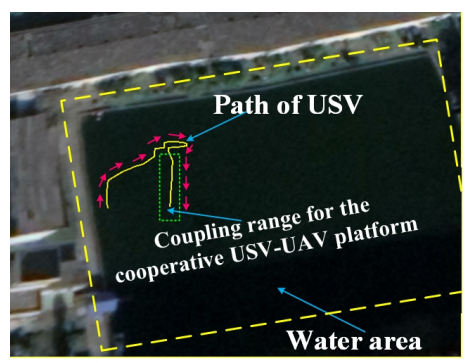

Fig. 8. Coupling process of the cooperative UAV-USV platform.

To accommodate the positioning accuracy, integrated ultrasonic sensors are adopted in the sensor system. When it comes to parameter performance of the selected sensors, the transmission center frequency is $40 \mathrm{kHz}$, the maximum transmission distance is $12 \mathrm{~m}$, the beam angle falls in $\left[50^{\circ}\right.$, $60^{\circ}$ ], and the precision of distance measuring is $1 \mathrm{~cm}$. As shown in Fig. 9, four ultrasonic sensors with vertical upward transmitting directions, are mounted on the front part of the USVs deck. Wherein two sensors are located on the port side at a distance of $1.5 \mathrm{~m}$ along $X$-direction of the UAV carrying platform, and at a transverse distance of $1 \mathrm{~m}$ along $Y$-direction, another two are located on the starboard side symmetrically.

To verify the effectiveness of the multi-ultrasonic joint dynamic positioning algorithm, a series of dynamic positioning experiments are executed by employing the integrated ultrasonic sensors. Four ultrasonic sensors $S_{u_{-} i}(i=1, \ldots, 4)$ obtain a sum of distance data when UAV landing on its carrying platform. And nine sets of randomly selected data achieved by sensors are listed in Table II.

Following that, with the aid of our multi-ultrasonic joint

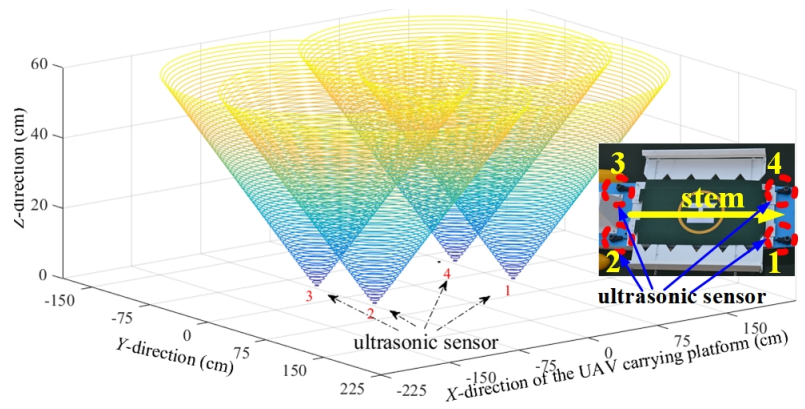

Fig. 9. Position distribution of ultrasonic sensors.

TABLE II

THE POSITION OF THE UAV SENSED BY THE MULTI-ULTRASONIC SENSOR SYSTEM (CM)

\begin{tabular}{|c|c|c|c|c|c|}
\hline & 1 & 2 & 3 & 4 & 5 \\
\hline$S_{u-1}$ & 462.31 & 431.90 & 461.71 & 413.91 & 512 \\
\hline$S_{u}-2$ & 480.40 & 494.91 & 544.03 & 435.10 & 494.51 \\
\hline$S_{u} 3$ & 535.82 & 523.93 & 551.10 & 432.32 & 445.62 \\
\hline$S_{u}-4$ & 514.41 & 468.50 & 467.56 & 409.85 & 461.93 \\
\hline & 6 & 7 & 8 & 9 & \\
\hline $\bar{S} S_{u \quad 1}$ & 527.81 & 487 & 438.50 & 582.54 & \\
\hline$S_{u_{-}-2}^{-}$ & 477.90 & 422.90 & 413 & 595.93 & \\
\hline$S_{u}^{-3}$ & 432 & 432.51 & 409.4 & 532.72 & \\
\hline$S_{u}^{-} 4$ & 494.81 & 491.33 & 436.22 & 513.31 & \\
\hline
\end{tabular}

dynamic positioning algorithm, four sets of position details $\left(x^{\prime}, y^{\prime}, z^{\prime}\right)$ of the UAV are reached. Wherein each set of the UAV's position is achieved by a data combination of three ultrasonic sensors $S_{u_{-} i j k}(i, j, k=1, \ldots, 4, i \neq j \neq k)$, and consists of nine points. Referring to details of the coupling

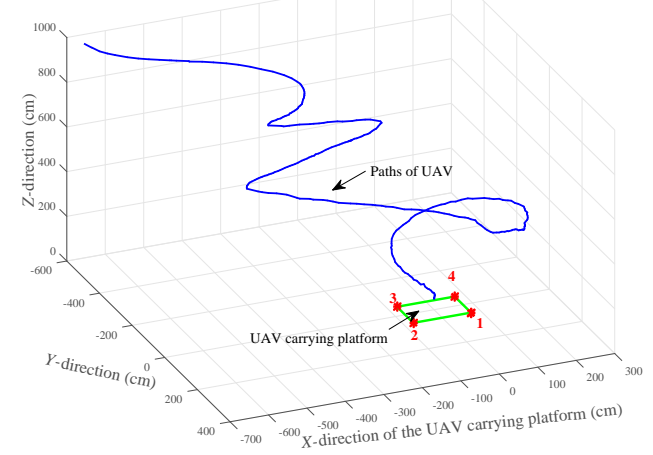

Fig. 10. Paths of UAV during the landing process in the plane of $X_{E S} O Y_{E S} Z_{E S}$ of the coupled USV-UAV systems.

experiments, in Fig. 8, path of USV in yellow color and the coupling range of the cooperative USV-UAV platform are presented vividly. Fig. 10 presents paths of UAV during the landing process. The time from USV received UAV landing request to UAV completely landed on the deck of USV is $3.6 \mathrm{~min}$, the time of the poses adjustment of USV and USV for landing is almost $2 \mathrm{~min}$, the time from UAV captured in the ultrasonic range for the first time to UAV completely landed on the deck of USV is near 1.6min. Velocities of UAV and USV are changing dynamically. Average velocities of USV and UAV are $0.45 \mathrm{~m} / \mathrm{s}$ and $0.26 \mathrm{~m} / \mathrm{s}$, respectively. Duration navigation distances of USV and UAV are $44.5 \mathrm{~m}$ and $25.6 \mathrm{~m}$, respectively. 
In Fig. 11(a), the UAV is over-flying the USV, and they cooperatively execute some task together at close range, such as surveillance, patrol, etc. After completing the deployed task, the UAV tends to land on its carrying platform and sends the landing commands to the USV. Accordingly, in Fig. 11(b), the USV unfolds the retractable UAV extended deck, and transmits the allowable landing commands to the UAV. By employing the multi-ultrasonic joint dynamic positioning algorithm, the dynamic position of the UAV can be achieved. When entering into the definite ultrasonic range, the UAV obtains a series of guide points by using our hierarchical landing guide point generation algorithm. Following that, with the aid of the above guide points, a sequence of cubic B-spline paths are generated for the UAV, and then UAV approaches to the USV safely and steadily as shown in Fig. 11(c). Eventually, in Fig. 11(d), the UAV lands on its carrying platform that located on the USV, and the retractable UAV extended deck is folded. The above experiments verify that the designed cooperative USV-UAV platform can undertake tasks cooperatively, and their coupled USV-UAV systems are effective.

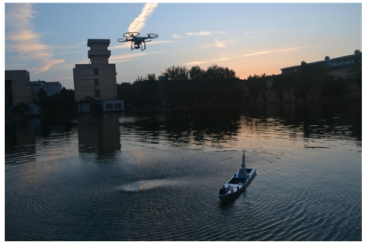

(a) USV-UAV systems cooperation.

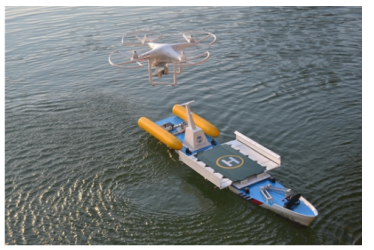

(c) UAV is flying to the USV.

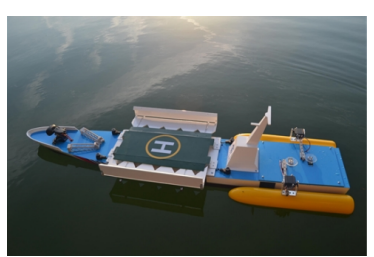

(b) USV unfolds for UAV landing.

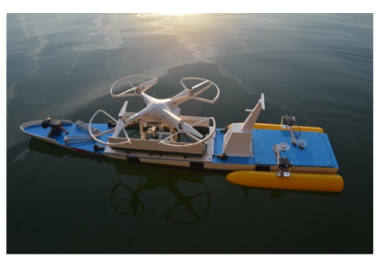

(d) UAV lands on the USV.
Fig. 11. Experiments of the coupled USV-UAV systems.

To validate the effectiveness of our coupled UAV-USV platform, a large number of water tests under generous of scenarios are carried out on the cooperative platform, including USV navigation test, UAV flight test and UAV-USV positioning test. Owing to the disturbances of the dynamic external environment, the landing test would be failed sometimes, including the UAV positioning test, the UAV tracking path test, and the UAV initial position capture. To resolve the above problems occurred in the UAV landing test, according to the mission planning arrangement, UAV flies to a set altitude to reland on its carrying platform. The given altitude should be fall in the maximum transmission distance of the selected sensors, and then should be less than $12 \mathrm{~m}$. Generally, the current success rate is quite high and almost $100 \%$ if the re-land procedure is introduced. And the average tests time of $3.6 \mathrm{~min}$.
Even occurring the dynamic environmental disturbances, with the aid of the UAV re-land procedure, as shown in Fig. 11(d) the UAV would also complete the landing in 10 minutes in general.

\section{Conclusions}

This paper investigates cooperative platform design for the coupled USV-UAV systems. We briefly state the cooperative USV-UAV platform, including its system architecture, transport security design of USV for UAV, and control system. Then, to achieve the position of UAV in real time, based on ToA, we propose a multi-ultrasonic joint dynamic positioning algorithm. To guarantee UAV landing on the USV steadily and successfully, a hierarchical landing guide point generation algorithm is introduced. And ways to plan smooth paths represented by the cubic B-spline curves are demonstrated. Simulations and experiments validate the effectiveness of our cooperative USV-UAV platform. In the near future, referring to the coupled USV-UAV systems, it is meaningful that, their internal cooperative mechanism and motion environments would be studied thoroughly. And the obstacle-avoidance path planning for the heterogeneous USV-UAV platform should be stressed in depth.

\section{REFERENCES}

[1] Y. Ma, H. Wang, and M. Zamirian, "A novel approach for multiple mobile objects path planning: Parametrization method and conflict resolution strategy," Phys. Lett. A, vol. 376, no. 4, pp. 377-386, 2012.

[2] Y. Ma, H. Wang, Y. Xie, and M. Guo, "Path planning for multiple mobile robots under double-warehouse," Inform. Sci., vol. 278, pp. 35779, 2014.

[3] Y.-L. Wang and Q.-L. Han, "Network-based fault detection filter and controller coordinated design for unmanned surface vehicles in network environments," IEEE Trans. Ind. Inform., vol. 12, no. 5, pp. 1753-1765, 2016.

[4] Y. Ma, M. Hu, and X. Yan, "Multi-objective path planning for unmanned surface vehicle with currents effects," ISA Trans., vol. 75, pp. 137-156, 2018.

[5] Y. Tang, H. Gao, J. Kurths, and J.-a. Fang, "Evolutionary pinning control and its application in uav coordination," IEEE Trans. Ind. Inform., vol. 8, no. 4, pp. 828-838, 2012.

[6] V. Roberge, M. Tarbouchi, and G. Labonté, "Comparison of parallel genetic algorithm and particle swarm optimization for real-time uav path planning," IEEE Trans. Ind. Inform., vol. 9, no. 1, pp. 132-141, 2013.

[7] L. R. Pinto, A. Moreira, L. Almeida, and A. Rowe, "Characterizing multihop aerial networks of cots multirotors," IEEE Trans. Ind. Inform., vol. 13, no. 2, pp. 898-906, 2017.

[8] Y. Ma, Y. Zhao, X. Qi, Y. Zheng, and R. Gan, "Cooperative communication framework design for the unmanned aerial vehicles-unmanned surface vehicles formation," Adv. Mech. Eng., vol. 10, no. 5, pp. 1-9, 2018.

[9] R. R. Murphy, E. Steimle, C. Griffin, C. Cullins, M. Hall, and K. Pratt, "Cooperative use of unmanned sea surface and micro aerial vehicles at hurricane wilma," J. Field Robot., vol. 25, no. 3, pp. 164-180, 2008.

[10] Y. Liu, W. Liu, R. Song, and R. Bucknall, "Predictive navigation of unmanned surface vehicles in a dynamic maritime environment when using the fast marching method," Int. J. Adapt. Contr. Signal Process., vol. 31, no. 4, pp. 464-488, 2017.

[11] Z. Liu, Y. Zhang, X. Yu, and C. Yuan, "Unmanned surface vehicles: An overview of developments and challenges," Аnnu. Rev. Contr., vol. 41, pp. 71-93, 2016. 
[12] B. Grocholsky, J. Keller, V. Kumar, and G. Pappas, "Cooperative air and ground surveillance," IEEE Robot. $\mathcal{E}$ Autom. Mag., vol. 13, no. 3, pp. 16-25, 2006.

[13] F. Muttin, "Umbilical deployment modeling for tethered uav detecting oil pollution from ship," Appl. Ocean Res., vol. 33, no. 4, pp. 332-343, 2011.

[14] N. Mathew, S. L. Smith, and S. L. Waslander, "Planning paths for package delivery in heterogeneous multirobot teams," IEEE Trans. Autom. Sci. Eng., vol. 12, no. 4, pp. 1298-1308, 2015.

[15] G. Wu, W. Pedrycz, H. Li, M. Ma, and J. Liu, "Coordinated planning of heterogeneous earth observation resources," IEEE Trans. Syst., Man, Cybern.: Syst., vol. 46, no. 1, pp. 109-125, 2016.

[16] Y. Chen and H.-L. Liu, "Overview of landmarks for autonomous, visionbased landing of unmanned helicopters," IEEE Aerospace and Electronic Systems Magazine, vol. 31, no. 5, pp. 14-27, 2016.

[17] L. Li, M. Yang, C. Wang, and B. Wang, "Hybrid filtering framework based robust localization for industrial vehicles," IEEE Transactions on Industrial Informatics, vol. 14, no. 3, pp. 941-950, 2018.

[18] Y. Kim, J. An, and J. Lee, "Robust navigational system for a transporter using gps/ins fusion," IEEE Transactions on Industrial Electronics, vol. 65 , no. 4 , pp. 3346-3354, 2018.

[19] V. A. Omar, "Modelling and control of a uav-usv collaboration scheme for fluvial operations," B.S. thesis, Universidad Carlos III de Madrid, Spain, 2017.

[20] S. Young, J. Peschel, G. Penny, S. Thompson, and V. Srinivasan, "Robotassisted measurement for hydrologic understanding in data sparse regions," Water, vol. 9, no. 7, p. 494, 2017.

[21] Y. Ma, Y. Zhao, J. Diao, L. Gan, H. Bi, and J. Zhao, "Design of sailassisted unmanned surface vehicle intelligent control system," Math. Probl. Eng., vol. 2016, pp. 1-13, 2016.

[22] G. Zhang and X. Zhang, "A novel dvs guidance principle and robust adaptive path-following control for underactuated ships using low frequency gain-learning,” ISA Trans., vol. 56, pp. 75-85, 2015.

[23] T. Van Haute, B. Verbeke, E. De Poorter, and I. Moerman, "Optimizing time-of-arrival localization solutions for challenging industrial environments," IEEE Trans. Ind. Inform., vol. 13, no. 3, pp. 1430-1439, 2017. 